\title{
Test beam operation of the CMS calorimeter trigger synchronization boards
}

\author{
A. David, ${ }^{a *}$ N. Almeida, ${ }^{a}$ J. da Silva, ${ }^{a}$ P. Silva ${ }^{a}$ and J. Varela ${ }^{a b}$ \\ ${ }^{a}$ LIP - Lisbon, \\ Av. Elias Garcia 14, 1000-149 Lisboa, Portugal \\ ${ }^{b} C E R N$, \\ 1221 Genève 23, Switzerland \\ E-mail: Andre.David@cern.ch
}

ABSTRACT: The CMS experiment uses information from its electromagnetic and hadronic calorimeters and muon detectors to decide whether to read out the whole detector. For such a task to be successful, all trigger primitives pushed through the trigger decision tree must be flawlessly aligned in time for operation at $40 \mathrm{MHz}$. Both calorimeters in CMS use the Synchronization Link Board for this purpose. In this article we report on the test results of this board using the bunched beams available in the $\mathrm{H} 4$ electron beam line at CERN.

KEYWORDS: Calorimeters; Data acquisition concepts; Modular electronics; Trigger concepts and systems (hardware and software).

${ }^{*}$ Corresponding author. 


\section{Contents}

1. Introduction 1

2. CMS Level-1 Trigger 1

3. The SLB test setup 3

4. Results

4.1 Beam time structure

4.2 Energy threshold settings

4.3 Accumulators in neighboring trigger tower 7

5. Conclusions 8

\section{Introduction}

The trigger system for the Compact Muon Solenoid (CMS) experiment at the CERN LHC will have to select 100 interesting events out of 32 million bunch crossings taking place every second [1]], each one with an average 20 interactions piled up.

This is achieved using a two-level architecture. The Level-1 (L1) Trigger reduces the event rate down to $100 \mathrm{kHz}$ using coarse information from the calorimeter and muon systems, by selecting signatures of muons, electrons, photons, jets and missing energy. Then the High Level Trigger (HLT) performs the final selection of events, reconstructing detailed information as needed, including hits in the silicon tracker in a commodity PC farm.

This article briefly reviews the Level-1 Trigger architecture of CMS and then focuses on results from the first bunched beam operation, at the CERN SPS, of the Synchronization Link Boards (SLB) connecting the Electromagnetic and Hadronic calorimeters (ECAL and HCAL) to the Regional Calorimeter Trigger (RCT).

\section{CMS Level-1 Trigger}

The CMS L1 Trigger [1] can be broadly divided into two branches, as depicted in figure 1. One of these is the Calorimeter branch, where data from the ECAL and HCAL detectors have to be combined by the RCT. After the RCT processing, the Global Calorimeter Trigger (GCT) calculates missing transverse energy and all interesting trigger candidate objects derived from calorimeter information (electron, photon and jet candidates).

Because of the high rates involved, the trigger and data acquisition systems are pipelined. All systems use the same clock derived from the LHC acceleration system, which also sets the 


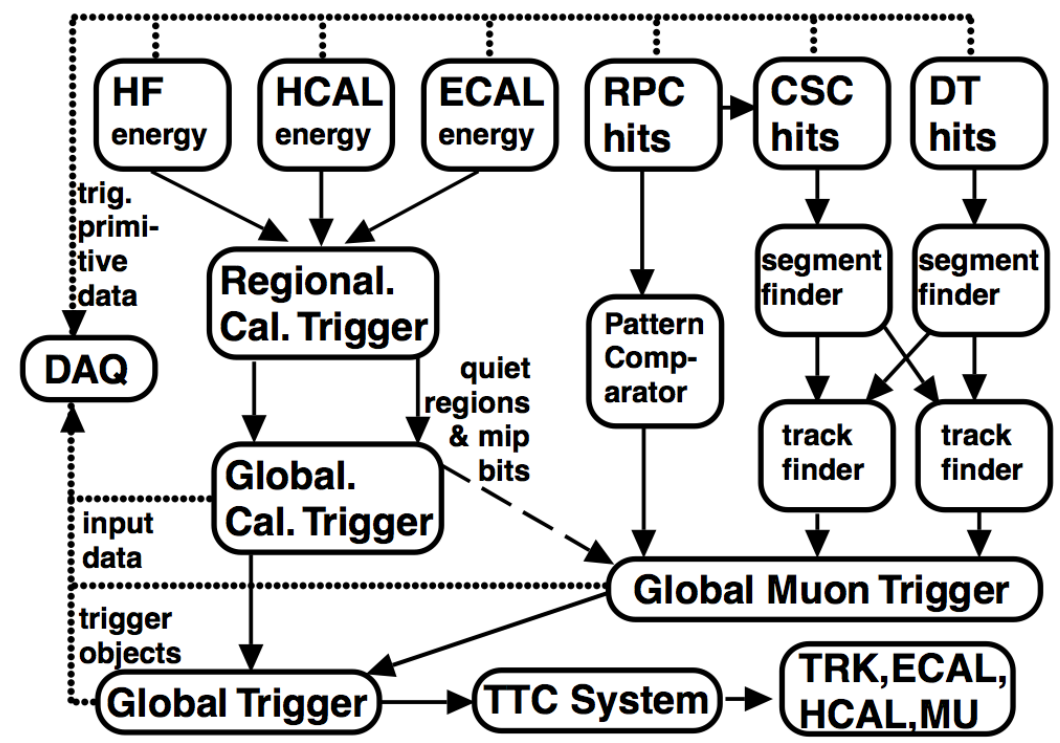

Figure 1. Overview of the CMS Level-1 trigger system. On the left the Calorimeter branch and on the right the Muon branch. Merging of data from different Calorimeter trigger data sources at the Regional Calorimeter Trigger has to be properly synchronized, which is accomplished using the SLB [2]牙].

beam structure, but because of cable and internal delays, different parts of the detector will be out of phase with respect to each other. Since the different detectors and the trigger might have different delays, the trigger data being pushed by sub-detectors like the ECAL and HCAL need to be synchronized before entering to the Trigger system. For this purpose a method for calorimeter trigger synchronization was developed and a dedicated mezzanine card, the Synchronization Link Board (SLB), was designed [2-4].

The synchronization task encompasses two aspects: clock phase compensation and pipeline length equalization. Clock phase synchronization is achieved by having the SLB sit between the sub-detector and Trigger systems, its outputs being driven by the same clock as the RCT inputs. Pipeline length equalization is then achieved by delaying data in an output FIFO, whose latency is automatically determined by the difference in time of arrival of the orbit signals from the Trigger and the sub-detectors.

An important feature of the SLB for checking synchronization of the ECAL and HCAL with the RCT during runtime is its built-in timing accumulators. Each accumulator histograms the timing of the trigger data produced by a trigger channel with respect to the start of orbit. These accumulators are 1024 LHC bunch crossings long and each bin is 11-bit wide (counting up to 2047). The accumulators also have a variable threshold, such that it is possible to count only trigger primitives whose transverse energy is above a certain value.

A procedure based on the correlation of the patterns recorded in these accumulators, which will be used to synchronize the more than 7000 trigger data channels of both ECAL and HCAL is described in []. 

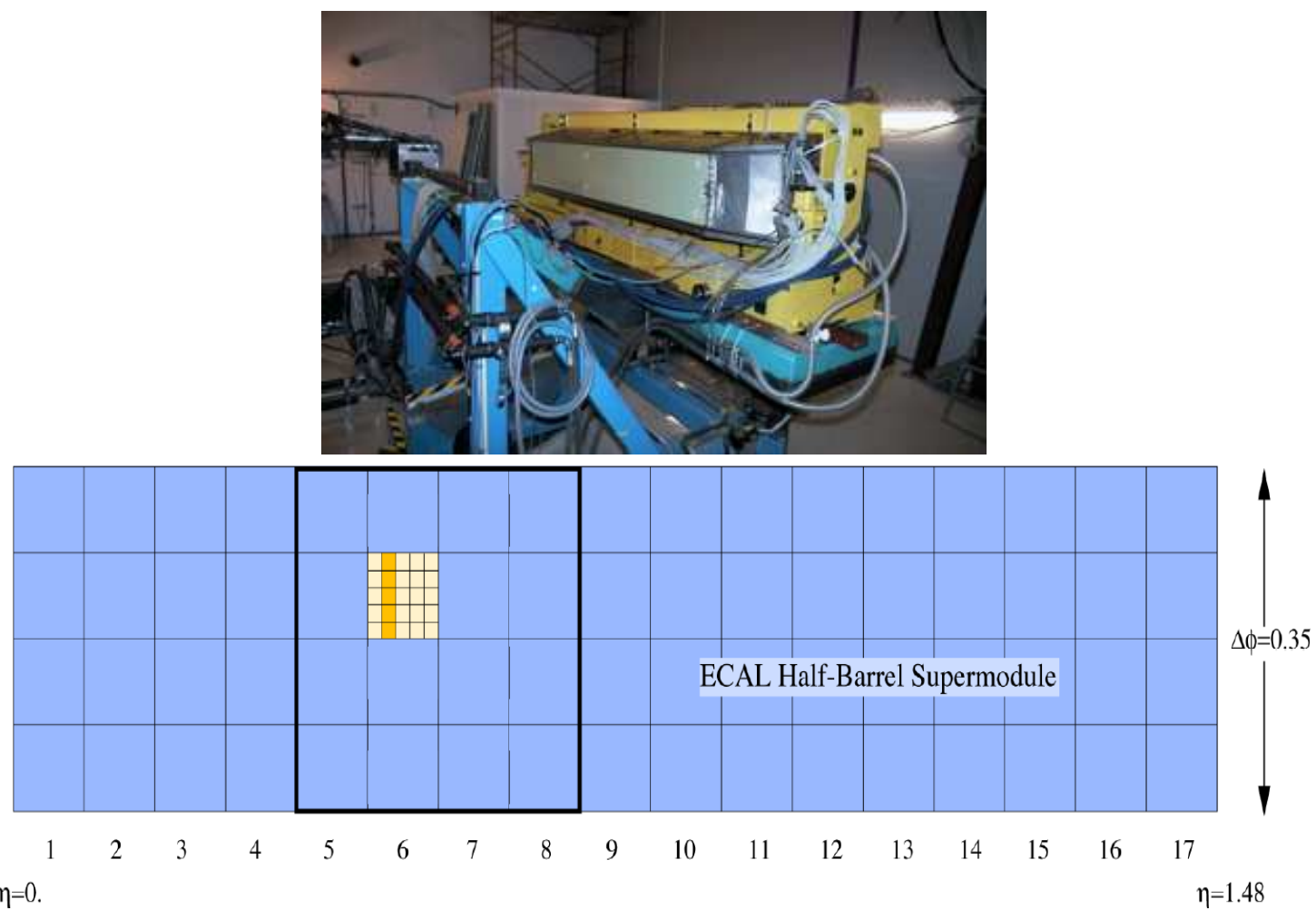

Figure 2. Top panel: physical view of an ECAL supermodule in the CERN SPS H4 beamline, where the electron beam comes from the left. Bottom panel: logical layout of an ECAL half-barrel supermodule, showing the 68 trigger towers, each merging information from 25 lead-tungstate crystals.

\section{The SLB test setup}

In order to validate the SLB cards, in particular its synchronization functionalities, we have used the CMS ECAL Collaboration Test Beam stand at the CERN SPS H4 beam line. Figure 2 (top) shows an ECAL barrel supermodule in the $\mathrm{H} 4$ beam area stand. The electron beam momentum is tunable between 10 and $120 \mathrm{GeV} / \mathrm{c}$. Results reported in this article correspond to data taken with a beam momentum of $50 \mathrm{GeV} / \mathrm{c}$, for which the beam spot $1 \sigma$ contour covers only one supermodule crystal.

Figure 目(bottom) shows the structure of an ECAL barrel supermodule which comprises 1700 lead-tungstate crystals grouped into 68 trigger towers (TT) of 25 crystals each. It is the data from each TT that are sent through the trigger path and synchronized and accumulated by the SLB. Trigger data are generated by adding, in the front-end electronics, the energy of the 25 crystals. Whenever there is a positive trigger decision, the individual crystal data are then read out by data concentrator cards. The readout system used at the $\mathrm{H} 4$ test beam is described in [5].

In order to test the operation of the accumulator histograms - the crucial part for monitoring the bunch synchronization of the calorimeter trigger data - we have taken data with the ECAL barrel final readout electronics, including the SLB cards, during a week when the SPS proton beam was bunched in a $25 \mathrm{~ns}$ LHC-like structure. For this, every SPS orbit (924 bunches long) nominally had one train of 48 bunches with beam. Figure 3 shows a measurement of the beam current as a 


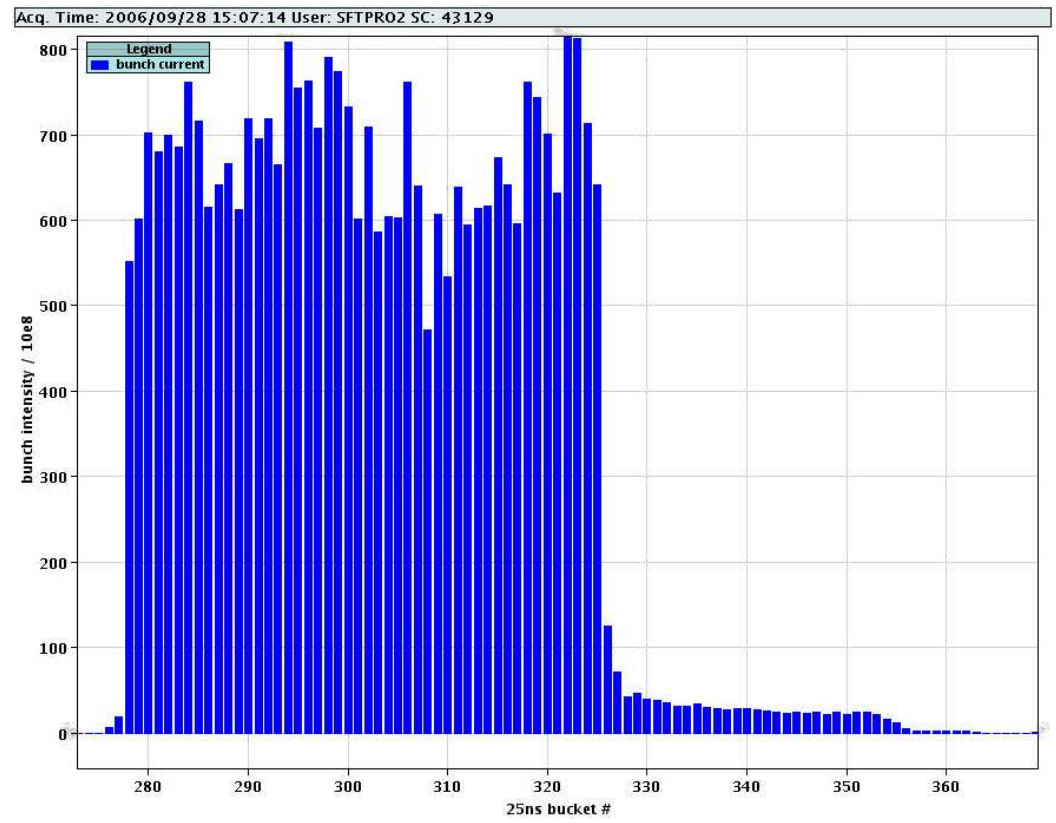

Figure 3. SPS bunched-beam timing structure as measured by the beam monitors. The beam bucket intensity is plotted as a function of the $25 \mathrm{~ns}$ bucket number, showing a non-trivial structure. (Courtesy of the CERN AB-OP group)

function of 25 ns "buckets", as provided by the CERN AB Department, which shows that there are also particles coming outside the nominal 48 bunch train.

\section{Results}

\subsection{Beam time structure}

The presence of the $25 \mathrm{~ns}$ structure in the beam timing pattern creates a distinctive feature in the SLB accumulators, which can record up to 1024 consecutive bunch crossings. The contents of one such accumulator is shown in figure 4 (top). There we see the same pattern repeated twice inside the 1024 bins. This was done on purpose to be able to make full use of the 1024 bins of the accumulators, profiting from the fact that the SPS orbit is shorter than that of the LHC. In order to quantify the delay between the similar patterns and the degree to which they are similar, we have calculated a sliding normalized self-correlation coefficient of the accumulator contents, using:

$$
C(\Delta b)=\frac{\sum_{i=\Delta b}^{1023}\left[A_{i}-\bar{A}_{\Delta b \cdots 1023}\right]\left[A_{i-\Delta b}-\bar{A}_{0 \cdots 1023-\Delta b}\right]}{\sqrt{\sum_{i=\Delta b}^{1023}\left[A_{i}-\bar{A}_{\Delta b \cdots 1023}\right]^{2} \sum_{i=\Delta b}^{1023}\left[A_{i-\Delta b}-\bar{A}_{0 \cdots 1023-\Delta b}\right]^{2}}},
$$

where $A_{i}$ represents the value of the accumulator in bin $i$ and $\bar{A}_{x \cdots y}=\frac{1}{y-x+1} \sum_{i=x}^{y} A_{i}$, is a simple average. This self-correlation is depicted in figure $\bigoplus$ (bottom) as a function of the delay, $\Delta b$. We can see that there is a trivial maximum for $\Delta b=0$, with $C(0)=1$. Furthermore, there is a second maximum at $\Delta b=924$ (insert figure4) exactly one SPS revolution period, with $C(924)=0.9971 \pm$ 0.007. For very large values of $\Delta b$ the number of bins contributing to the self-correlation is so small that the self-correlation values fluctuate significantly. 


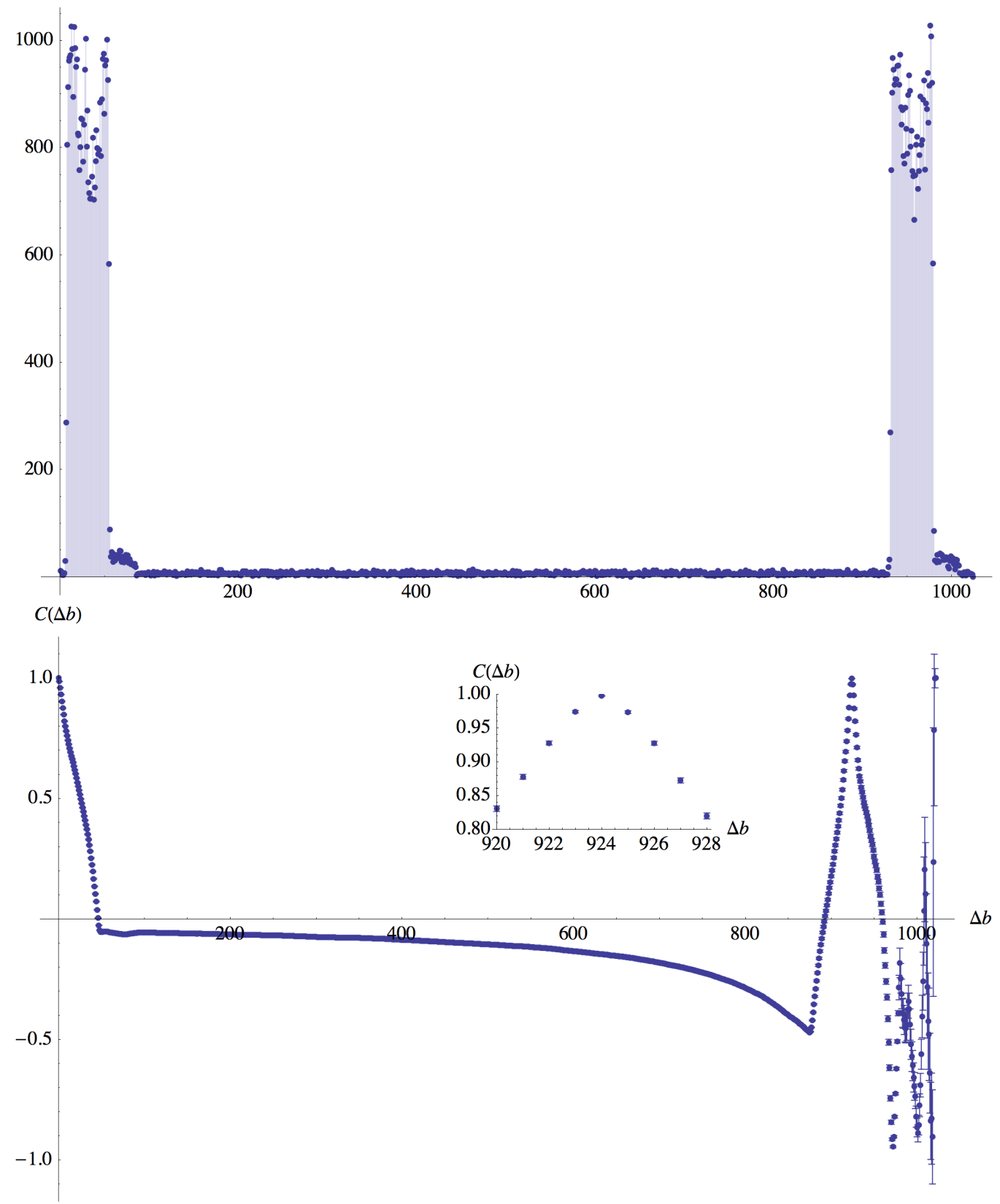

Figure 4. The top panel shows the contents of the SLB accumulator histogram for the beam structure depicted in figure 3. The bottom panel plots the self-correlation which shows a non trivial maximum at a delay of 924 bunch crossings (inset,) or a full SPS orbit period.

In order to quantify the precision with which the timing of the second maximum is extracted, we can look at the weighted distance between the self-correlation value at the maximum and its closest neighbors: $D_{1}(\Delta b)=\operatorname{Min}\left\{|C(\Delta b)-C(\Delta b \pm 1)| / \sigma_{C(\Delta b)-C(\Delta b \pm 1)}\right\}$, where the calculation of $\sigma_{C(\Delta b)-C(\Delta b \pm 1)}$ takes into account the correlations between the individual self-correlation values. This distance can be seen as a significance or how many "sigmas" the values are away from each other. Using the accumulator statistics shown in figure 4 , we find $D_{1}(924)=13.1$. The significance 


\section{Bunch structure measured at TT=59 by SLB 742}

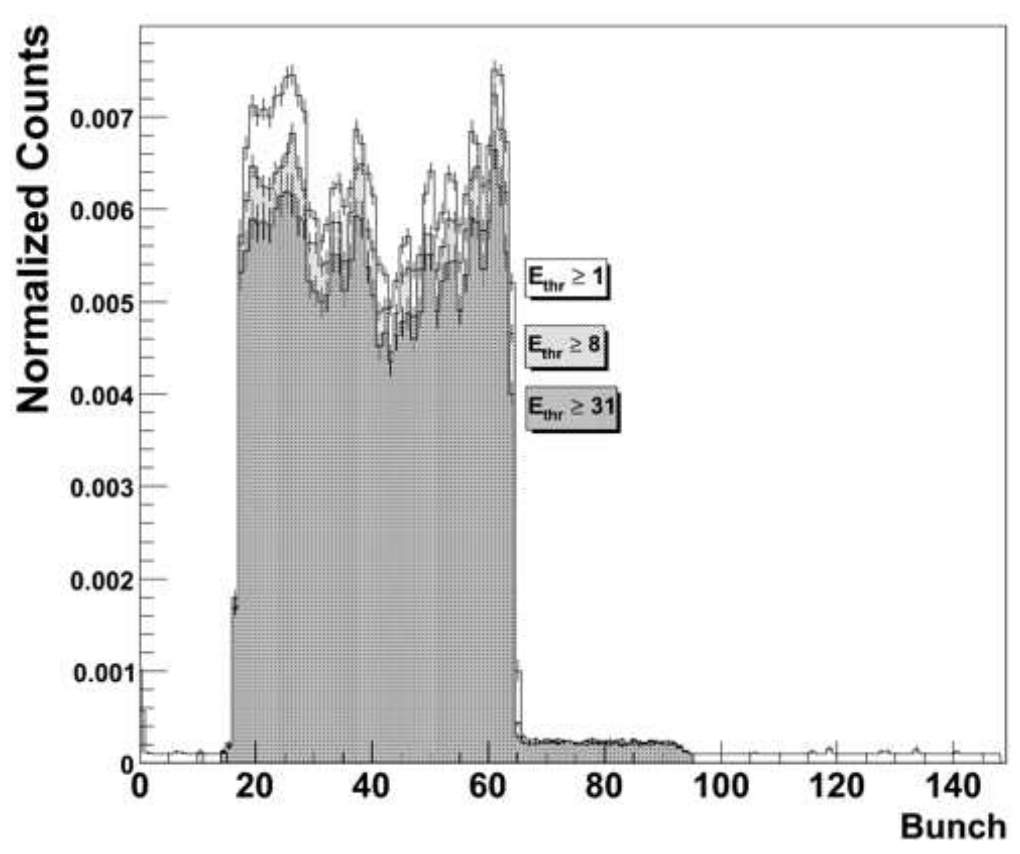

Figure 5. Contents of an SLB accumulator normalized to the intensity of the beam when different energy thresholds are applied. Note that going from 1 to 8 in the energy threshold, the noise before 15 and after 95 bunch crossings is completely removed.

can be translated into an estimation of the error in $\Delta \mathrm{b}$ for which the maximum occurs using $\sigma_{\Delta b}=$ $1 / D_{1}(\Delta b)$. We obtain $\Delta b=924.00 \pm 0.08$ for the second maximum in the self-correlation function.

A discussion of this timing method in the context of the LHC operation is presented in reference [П]. The precision is dependent of the accumulated statistics and trigger data alignment is considered to be obtained if the significance is greater than five. At LHC low luminosity $\left(10^{32} \mathrm{~cm}^{-2} \mathrm{~s}^{-1}\right)$, this is achieved per trigger tower after few minutes of LHC running 佨.

\subsection{Energy threshold settings}

The next interesting quantity that can be studied is the amount of trigger primitives accumulated for different energy threshold values. For this study, we have taken data with different energy thresholds applied to a certain SLB accumulator and then compared their contents by normalizing them to the integrated beam intensity. Figure 5 shows the effect of applying different threshold values to the trigger primitives contributing to the accumulators. The threshold values are defined units of $160 \mathrm{MeV}$.

When comparing the accumulator contents with the threshold at 1 and $8(160$ and $1280 \mathrm{MeV}$, respectively), one can easily see that the background before 15 and after 95 bunch crossings disappears. This means that for energy values below $160 \mathrm{MeV}$ there is still a considerable contamination from electronics noise. 

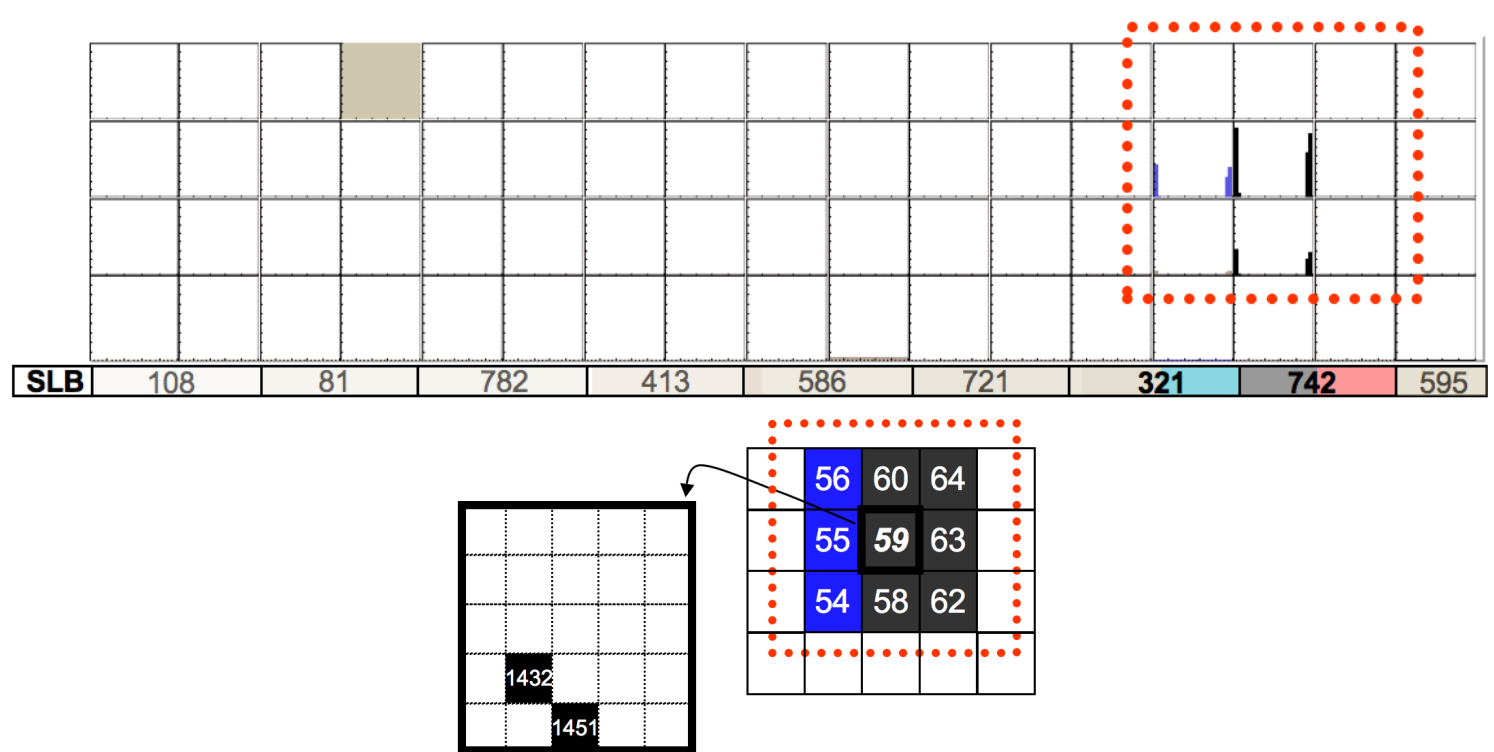

Figure 6. Layout of an ECAL barrel supermodule in terms of its trigger towers. In the top, the accumulator histograms for the 68 trigger towers, showing non-empty accumulators inside the dotted area, which was exposed to beam. One can also see the serial numbers of the SLB cards reading out 8 TT each, except for the last one. Bottom: trigger tower numbering for the region of interest. TT 59 in the center had crystals 1432 and 1451 irradiated during these studies. These correspond to a symmetric and an asymmetric position with respect to the neighboring trigger towers, 55 and 58.

\subsection{Accumulators in neighboring trigger tower}

The counts registered in the accumulators as a function of the energy threshold depend on the distribution of the energy deposits in the corresponding trigger towers.

The electron beam used in the SPS $\mathrm{H} 4$ beam line has, at $50 \mathrm{GeV} / \mathrm{c}$, a spatial dispersion of the order of a crystal's size. On the other hand more than $90 \%$ of the electromagnetic shower energy is contained in a $3 \times 3$ crystal matrix. In consequence, when the beam in centered in crystals close to the boundary between two trigger towers, the electron energy deposit is shared between the two towers. For each event, the fraction of this energy sharing depends on the electron impact coordinates.

Therefore, when the beam is pointing to a crystal close to the tower's boundary, it is expected to observe counts in the SLB accumulators of the towers neighbor to the central tower (the tower where the beam is impinging). This can be seen in figure 6 (top), where the towers left and below of the central one also show some activity.

For this study we chose a specific trigger tower (59) and centered the beam in 2 crystals, corresponding to a symmetric (crystal 1432) and an asymmetric (crystal 1451) position relative to the neighboring towers (55 and 58) as shown in figure 6 (bottom).

With the beam centered in crystal 1432 we expect that the number of counts in TT55 and TT58 to be the roughly the same. Figure 7 (left) plots the ratio of the number of counts in the neighboring towers (55 and 58) to the central tower (59), as a function of energy threshold up to about $5 \mathrm{GeV}$. The dependence on the energy threshold is similar in both cases reflecting symmetri- 

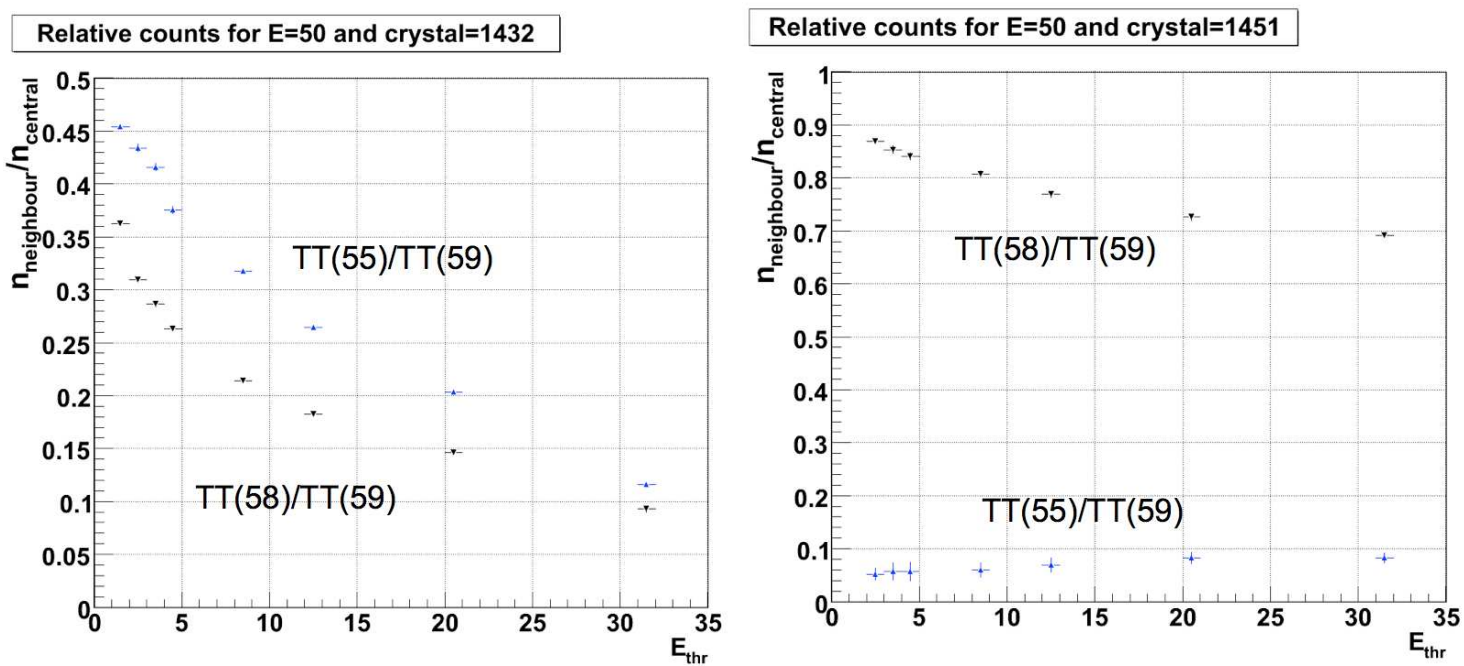

Figure 7. Evolution of the ratio of counts registered in neighboring towers (55 and 58) with respect to the number of counts registered in the tower (59) in which the beam is centered for different energy thresholds applied to the SLB accumulators. Left: the case in which the irradiated crystal is equally distant from the neighboring towers. Right: the case in which the crystal is closer to trigger tower 58.

cal energy leakage in the two neighbor towers. The counts in the neighbor towers are at the level of $40 \%$ of those of the central tower, for the lowest threshold. This can be explained by the fact that the neighbor towers are outside the $3 \times 3$ window of shower containment for electrons hitting the center of crystal 1432, and therefore are only sensitive to electrons with impact coordinates biased towards those towers. The fact that the two ratios are not at the exact same level (45\% and 35\%) for the lowest threshold can be due to an asymmetry of the beam profile (larger dispersion in the horizontal direction).

When centering the beam on crystal 1451 , which is a close neighbor to TT58 and is two crystals away from the TT55 boundary, we expect a large number of counts in TT58 and the number of counts in TT55 to be heavily suppressed. This is shown in figure 7 (right). The fact that the ratio T55/T59 is independent of the energy threshold is indicative that in this case the T55 counts might be due to beam bremsstrahlung or to particles in the tails of the horizontal beam profile distribution.

\section{Conclusions}

The operation of the trigger link synchronization boards for the CMS calorimeters has been successfully validated in the bunched electron beam test setup at the SPS H4 beam line. The accumulators in these boards have a crucial role in monitoring the trigger data bunch synchronization in the calorimeter trigger of CMS.

\section{Acknowledgments}

We would like to express our gratitude to all the members of the CMS ECAL Collaboration who have helped in the data taking campaign at $\mathrm{H} 4$ and to the CERN AB-OP group for making beam intensity plots available for comparison with our data. 


\section{References}

[1] CMS collaboration, The TRIDAS Project Technical Design Report, Volume 1: The Level-1 Trigger, CERN/LHCC 2000-38, CMS TDR 6.1, http://cmsdoc.cern.ch/cms/TDR/TRIGGER-public/trigger.htm.

[2] N. Almeida, J.C. Da Silva, R. Alemany and J. Varela, Calorimeter Trigger Synchronization in CMS, Implementation and Test System, $10^{\text {th }}$ Workshop on Electronics for LHC Experiments and Future Experiments, Boston U.S.A., September 2004, CMS CR-2004/068.

[3] N. Almeida, J. C. Silva and J. Varela, Description of the synchronization and link board. ECAL and HCAL interface to the regional calorimeter trigger, CMS IN-2005/007.

[4] N. Almeida et al., Calorimeter trigger synchronization in the CMS experiment, Nucl. Instrum. Meth $\mathbf{5 6 8 ( 2 0 0 6 ) 6 3 4 .}$

[5] P. Musella et al., The CMS Electromagnetic Calorimeter data acquisition system at the 2006 test beam, $15^{\text {th }}$ IEEE-NPSS Real-Time Conference, Batavia U.S.A., 29 April - 4 May 2007, RT2007-TD-DAQ01. 